\title{
Methyl 4-Pentafluorosulfanylphenyl Sulfoximines
}

\author{
Christine M. M. Hendriks \\ Jens Reball \\ Carsten Bolm* \\ Institute of Organic Chemistry, RWTH Aachen University, Landoltweg 1, 52074 Aachen, Germany \\ carsten.bolm@oc.rwth-aachen.de
}

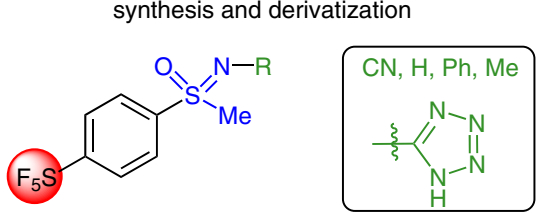

Received: 26.09.2014

Accepted after revision: 20.10 .2014

Published online: 20.11 .2014

DOI: 10.1055/s-0034-1378936; Art ID: st-2014-b0809-c

Abstract A low-cost and high-yielding synthetic route towards methyl 4-pentafluorosulfanylphenyl sulfoximines from the corresponding sulfide has been developed. The intermediate $\mathrm{N}$-cyano sulfoximine was converted into the corresponding $\mathrm{N}$ - $(1 \mathrm{H})$-tetrazole, and the $\mathrm{NH}$-sulfoximine was modified by $\mathrm{N}$-arylation and $\mathrm{N}$-alkylation reactions.

Key words building blocks, pentafluorosulfanyl group, sulfoximine, organofluorine compounds, sulfilimine

Fluorine-containing compounds exhibit unique physicochemical properties and, consequently, they are of interest in medicinal chemistry, crop protection, and material sciences. ${ }^{1}$ In this context, the pentafluorosulfanyl group $\left(\mathrm{SF}_{5}\right)$, also known as 'super-trifluoromethyl' group, plays a very special role. ${ }^{1,2}$ Noteworthy are, for example, the high thermal stability of aryl sulfurpentafluorides and the chemical inertness of the $\mathrm{SF}_{5}$ group towards hydrolysis. ${ }^{3}$ Compared with a trifluoromethyl substituent, the $\mathrm{SF}_{5}$ group has a higher electronegativity ${ }^{4}$ and polarity, and the respective molecules show improved lipophilicity. ${ }^{5}$ As a result, the $\mathrm{SF}_{5}$ group has become an attractive structural motif in the design of biologically active compounds, ${ }^{2 b, c, 6}$ functional materials, ${ }^{7}$ and, as recently reported, in Brønsted acid catalysts. $^{8}$

Due to the fact that only a few efficient synthetic methods for the introduction of the $\mathrm{SF}_{5}$ group exist, ${ }^{3,9}$ commercially available $\mathrm{SF}_{5}$-containing building blocks are rare and most of them are expensive. Therefore, the development of new scaffolds with $\mathrm{SF}_{5}$ groups appears to be desirable.

Sulfoximines, the mono-aza analogues of sulfones, are widely used in asymmetric synthesis and catalysis. ${ }^{10}$ Especially in the last years, such compounds have also attracted attention as drugs ${ }^{11}$ and crop protection agents. ${ }^{12}$ Advantageously, in contrast to sulfones, they are modifiable at the sulfoximine nitrogen, which can lead to beneficial effects on the solubility of the respective molecules. ${ }^{13}$ Fluorinecontaining sulfoximines ${ }^{11,12,14-16}$ are of particular interest because they combine the advantages of the sulfoximidoyl moiety with the favorable electronic and steric properties induced by, for example, a fluoro or a trifluoromethyl substituent. However, to our knowledge, sulfoximines bearing $\mathrm{SF}_{5}$ groups are unprecedented. Here, we fill this synthetic gap and report on preparative routes towards a range of key compounds with sulfoximidoyl cores and $\mathrm{SF}_{5}$ substituents.

For the preparation of the first target molecule $(\mathrm{NH}-$ sulfoximine 4), methyl 4-pentafluorosulfanylphenyl sulfide (1) was regarded as a promising starting material. ${ }^{17}$ Fulfilling our expectations, the imination of 1 with cyanamide and $N$-bromosuccinimide (NBS) ${ }^{18}$ proceeded smoothly, affording the corresponding $N$-cyano sulfilimine 2 in $96 \%$ yield (Scheme 1). Subsequent oxidation with $m$-CPBA ${ }^{18}$ led to $\mathrm{N}$-cyano sulfoximine 3 in $81 \%$ yield. Finally, the $\mathrm{CN}$-group was cleaved upon treatment with $50 \%$ aq. $\mathrm{H}_{2} \mathrm{SO}_{4}$ at $110{ }^{\circ} \mathrm{C},{ }^{19}$ providing the desired $\mathrm{NH}$-sulfoximine 4 in $71 \%$ yield. ${ }^{20}$ Both enantiomers of the racemic mixture could be separated on analytical CSP HPLC, which allowed 4 to be obtained in non-racemic form by preparative HPLC separation. ${ }^{21}$

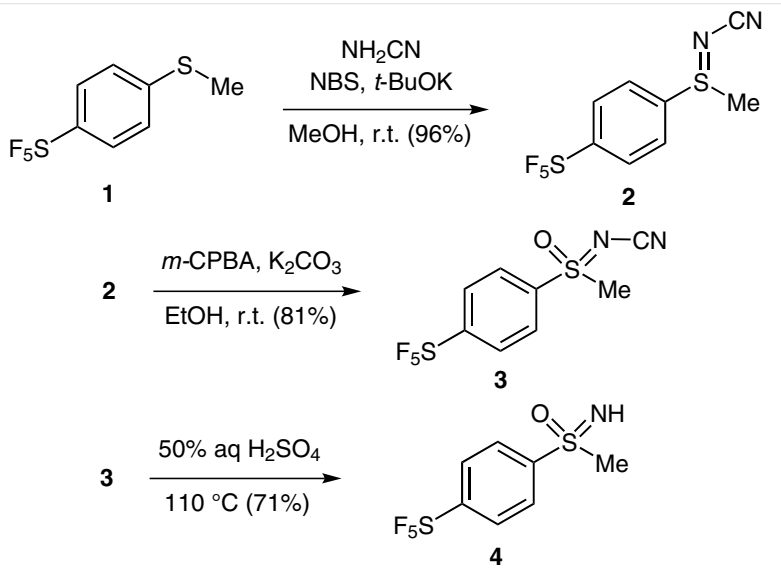

Scheme 1 Synthesis of methyl 4-pentafluorosulfanylphenyl sulfoximine 4

Considering that $\mathrm{N}$-arylated sulfoximines can be highly selective ligands in asymmetric metal catalysis, ${ }^{10,22}$ we first investigated the application of a representative N-phenylation protocol allowing the conversion of 4-pentafluorosulfanylphenyl sulfoximine (4) into $\mathrm{N}$-arylated sulfoximine $\mathbf{5}$ under copper catalysis. ${ }^{23}$ To our delight, this approach was 
highly efficient, providing $N$-phenyl sulfoximine $\mathbf{5}$ in $93 \%$ yield starting from $\mathbf{4}$ and iodobenzene as aryl source (Scheme 2).

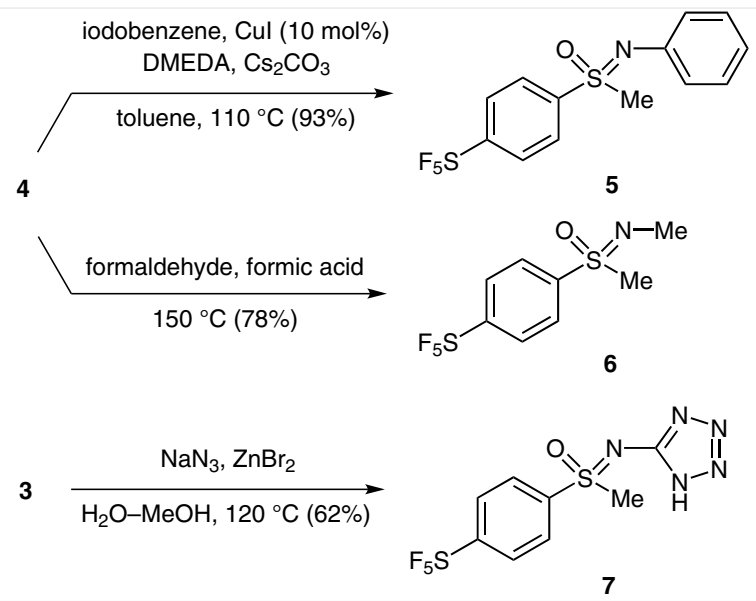

Scheme 2 Derivatizations of $\mathrm{NH}$-sulfoximine $\mathbf{4}$ and conversion of $\mathrm{N}$-cyano sulfoximine 3

We then focused on the N-methylation of $\mathbf{4}$ to give $\mathbf{6}$. This transformation was regarded as particularly important because it was recently demonstrated that several $\mathrm{N}$-methyl sulfoximines showed a significantly higher solubility compared with their isolipophilic counterparts in the sulfone series, ${ }^{13 a}$ leading to beneficial effects in their respective bioactivity studies. Here, the N-methylation of $\mathbf{4}$ was successfully performed under Eschweiler-Clark conditions, ${ }^{24,25}$ affording $\mathrm{N}$-methylated sulfoximine $\mathbf{6}$ in $78 \%$ yield (Scheme 2).

Considering that tetrazoles are carboxylic acid bioisosteres that often exhibit high bioactivities, ${ }^{26}$ the conversion of $\mathrm{N}$-cyano sulfoximine $\mathbf{3}$ into tetrazole $\mathbf{7}$ was studied. ${ }^{27}$

By using a combination of $\mathrm{NaN}_{3}$ and $\mathrm{ZnBr}_{2}$ in methanolwater, formation of the heterocycle proceeded smoothly, leading to $\mathrm{N}$-(1H)-tetrazole methyl 4-pentafluorosulfanylphenyl sulfoximine ( 7 ) in $62 \%$ yield (Scheme 2 ).

The three representative synthetic transformations depicted in Scheme 2 allow us to draw two significant conclusions: first, compounds such as $\mathbf{4}$ are readily available; and second, standard protocols can be used for modifications of sulfoximines with 4-pentafluorosulfanyl substituents providing interesting new building blocks for future synthetic and biological applications.

\section{Acknowledgment}

We thank Melissa Plag for various synthetic contributions and Prof. Dr. P. Kirsch, Merck KGaA, for highly stimulating discussions.

\section{Supporting Information}

Supporting information for this article is available online at http://dx.doi.org/10.1055/s-0034-1378936.

\section{References and Notes}

(1) For reviews, see: (a) Kirsch, P. Modern Fluoroorganic Chemistry; Wiley-VCH: Weinheim, 2004. (b) Crowley, P. J.; Mitchell, G.; Salmon, R.; Worthington, P. A. Chimia 2004, 58, 138. (c) Jeschke, P. ChemBioChem 2004, 5, 570. (d) Pursur, S.; Moore, P. R.; Swallow, S.; Gouverneur, V. Chem. Soc. Rev. 2008, 37, 320. (e) Fluorine in Medicinal Chemistry and Chemical Biology; Ojima, I., Ed.; Wiley-Blackwell: Chichester, 2009. (f) Ojima, I. J. Org. Chem. 2013, 78, 6358. (g) Wang, J.; Sánchez-Roselló, M.; Aceña, J. L.; Del Pozo, C.; Sorochinsky, A. E.; Fustero, S.; Soloshonok, V. A.; Liu, H. Chem. Rev. 2014, 114, 2432.

(2) (a) Sheppard, W. A. J. Am. Chem. Soc. 1960, 82, 4751. (b) Welch, J. T. In Fluorine in Pharmaceutical and Medicinal Chemistry; Vol. 6; Gouverneur, V.; Muellen, K., Eds.; Imperial College Press: London, 2012, 175. (c) Altomonte, S.; Zanda, M. J. Fluorine Chem. 2012, 143, 57. (d) Savoie, P. R.; Welch, J. T. Chem. Rev., DOI: 10.1021/cr500336u.

(3) Sheppard, W. A. J. Am. Chem. Soc. 1962, 84, 3064.

(4) Sheppard, W. A. J. Am. Chem. Soc. 1962, 84, 3072.

(5) Hansch, C.; Muir, R. M.; Fujita, T.; Maloney, P. P.; Geiger, F.; Streich, M. J. Am. Chem. Soc. 1963, 85, 2817.

(6) For recent examples, see: (a) Altomonte, S.; Baillie, G. L.; Ross, R. A.; Riley, J.; Zanda, M. RSC Adv. 2014, 4, 20164. (b) Chia, P. W.; Brennan, S. C.; Slawin, A. M. Z.; Riccardi, D.; O'Hagan, D. Org. Biomol. Chem. 2012, 10, 7922. (c) Micheli, F.; Andreotti, D.; Braggio, S.; Checchia, A. Bioorg. Med. Chem. Lett. 2010, 20, 4566. (d) Mo, T.; Mi, X.; Milner, E. E.; Dow, G. S.; Wipf, P. Tetrahedron Lett. 2010, 51, 5137. (e) Stump, B.; Eberle, C.; Schweizer, W. B.; Kaiser, M.; Brun, R.; Krauth-Siegel, R. L.; Lentz, D.; Diederich, F. ChemBioChem 2009, 10, 79. (f) Wipf, P.; Mo, T.; Geib, S. J.; Caridha, D.; Dow, G. S.; Gerena, L.; Roncal, N.; Milner, E. E. Org. Biomol. Chem. 2009, 7, 4163. (g) Lim, D. S.; Choi, J. S.; Pak, C. S.; Welch, J. T. J. Pestic. Sci. 2007, 32, 255.

(7) For examples, see: (a) Ye, C.; Gard, G. L.; Winter, R. W.; Syvret, R. G.; Twamley, B.; Shreeve, J. M. Org. Lett. 2007, 9, 3841. (b) Winner, S. W.; Winter, R. W.; Smith, J. A.; Gard, G. L.; Hannah, N. A.; Rananavare, S. B.; Piknova, B.; Hall, S. B. Mendeleev Commun. 2006, 16, 182. (c) Kirsch, P.; Hahn, A. Eur. J. Org. Chem. 2005, 3095.

(8) (a) Prévost, S.; Dupré, N.; Leutzsch, M.; Wang, Q.; Wakchaure, V.; List, B. Angew. Chem. Int. Ed. 2014, 53, 8770; Angew. Chem. 2014, 126, 8915. (b) Lee, J.-W.; List, B. J. Am. Chem. Soc. 2012, $134,18245$.

(9) (a) Bowden, R. D.; Greenhall, M. P.; Moillet, J. S.; Thomson, J. F2 Chemicals WO9705106, 1997; Chem. Abstr. 1997, 126, 199340. (b) Umemoto, T. IM\&T Research Inc. W02010014665 (A1), 2010.

(10) For overviews, see: (a) Johnson, C. R. Acc. Chem. Res. 1973, 6, 341. (b) Reggelin, M.; Zur, C. Synthesis 2000, 1. (c) Harmata, M. Chemtracts 2003, 16, 660. (d) Okamura, H.; Bolm, C. Chem. Lett. 2004, 33, 482. (e) Gais, H.-J. Heteroat. Chem. 2007, 18, 472. (f) Worch, C.; Mayer, A. C.; Bolm, C. In Organosulfur Chemistry in Asymmetric Synthesis; Torru, T.; Bolm, C., Eds.; Wiley-VCH: Weinheim, 2008, 209. (g) Bolm, C. Latv. J. Chem. 2012, 49. 
(11) For a recent review on sulfoximines in medicinal chemistry, see: Lücking, U. Angew. Chem. Int. Ed. 2013, 52, 9399; Angew. Chem. 2013, 125, 9570.

(12) For a recent review on sulfoxaflor and sulfoximines used as insecticides, see: Sparks, T. C.; Watson, G. B.; Loso, M. R.; Geng, C.; Babcock, J. M.; Thomas, J. D. Pestic. Biochem. Physiol. 2013, $107,1$.

(13) (a) Goldberg, F. W.; Kettle, J. G.; Xiong, J.; Lin, D. Tetrahedron 2014, 70, 6613. (b) Lücking, U.; Jautelat, R.; Krüger, M.; Brumby, T.; Lienau, P.; Schäfer, M.; Briem, H.; Schulze, J.; Hillisch, A.; Reichel, A.; Wengner, A. M.; Siemeister, G. ChemMedChem 2013, 8, 1067.

(14) For overviews on fluorinated sulfoximines, see: (a) Bizet, V.; Kowalczyk, R.; Bolm, C. Chem. Soc. Rev. 2014, 43, 2426. (b) Shen, X.; Hu, J. Eur. J. Org. Chem. 2014, 4437.

(15) For examples of bioactive 4-fluoroaryl sulfoximines, see: (a) Shetty, S. J.; Patel, G. D.; Lohray, B. B.; Chakrabarti, G.; Chatterjee, A.; Jain, M. R.; Patel, P. R. Cadila Healthcare WO2007077574 (A2), 2007. (b) Kahraman, M.; Sinishtaj, S.; Dolan, P. M.; Kensler, T. W.; Peleg, S.; Saha, U.; Chuang, S. S.; Bernstein, G.; Korczak, B.; Posner, G. H. J. Med. Chem. 2004, 47, 6854.

(16) For other selected contributions including 4-fluoro and 4-trifluoromethylaryl sulfoximines, see: (a) Kowalczyk, R.; Edmunds, A. J. F.; Hall, R. G.; Bolm, C. Org. Lett. 2011, 13, 768. (b) Barry, N.; Brondel, N.; Lawrence, S. E.; Maguire, A. R. Tetrahedron 2009, 65, 10660. (c) Terrier, F.; Magnier, E.; Kizilian, E.; Wakselman, C.; Buncel, E. J. Am. Chem. Soc. 2005, 127, 5563. (d) Reggelin, M.; Gerlach, M.; Vogt, M. Eur. J. Org. Chem. 1999, 1011.

(17) For the synthesis of 1 starting from 4-nitrophenyl sulfurpentafluoride, see: Beier, P.; Pastýř́íková, T.; Vida, N.; Iakobson, G. Org. Lett. 2011, 13, 1466.

(18) García Mancheño, O.; Bistri, O.; Bolm, C. Org. Lett. 2007, 9, 3809.

(19) Stoss, P.; Satzinger, G. Tetrahedron Lett. 1973, 267.

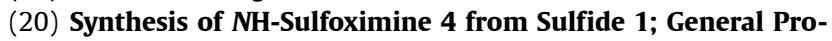
cedure: Step 1: To a solution of $\mathbf{1}(1.0 \mathrm{mmol})$ in $\mathrm{MeOH}(6 \mathrm{~mL})$, was added $\mathrm{NH}_{2} \mathrm{CN}$ (76 mg, $\left.1.8 \mathrm{mmol}\right), t$-BuOK $(191 \mathrm{mg}$, $1.7 \mathrm{mmol}$ ) and NBS (356 $\mathrm{mg}, 2.0 \mathrm{mmol}$ ). The reaction was stirred at room temperature until the starting material was consumed (reaction monitored by TLC). After removing the solvent under reduced pressure, water $(10 \mathrm{~mL})$ was added and the mixture was extracted with $\mathrm{CH}_{2} \mathrm{Cl}_{2}(3 \times 20 \mathrm{~mL})$. The combined organic layers were dried over anhydrous magnesium sulfate and the solvents were removed under reduced pressure. Purification by flash column chromatography provided $\mathrm{N}$-cyanosulfilimine 2. Step 2: To a solution of $\mathbf{2}(0.3 \mathrm{mmol})$ in $\mathrm{EtOH}(2.7 \mathrm{~mL})$ was added $m$-CPBA (ca. 70\%, $101 \mathrm{mg}, 0.45 \mathrm{mmol}$ ). The reaction mixture was stirred at room temperature until the starting material was consumed (reaction monitored by TLC). After removing the solvent under reduced pressure, $\mathrm{H}_{2} \mathrm{O}(5 \mathrm{~mL})$ was added and the mixture was extracted with $\mathrm{CH}_{2} \mathrm{Cl}_{2}(3 \times 10 \mathrm{~mL})$. The combined organic layers were dried over anhydrous magnesium sulfate and the solvents were removed under reduced pressure. Purification by flash column chromatography provided $N$-cyanosulfoximine 3. Step 3: A solution of $\mathbf{3}(0.23 \mathrm{mmol})$ in $50 \%$ aq $\mathrm{H}_{2} \mathrm{SO}_{4}(2.3 \mathrm{~mL})$ was stirred at $110{ }^{\circ} \mathrm{C}$ for $2 \mathrm{~h}$. After cooling to room temperature and adjusting the $\mathrm{pH}$ to 9 by addition of aq. sat $\mathrm{NaHCO}_{3}$ and aq. sat $\mathrm{NaOH}$ solution, the mixture was extracted with $\mathrm{CH}_{2} \mathrm{Cl}_{2}(3 \times 20 \mathrm{~mL})$. The combined organic layers were dried over anhydrous magnesium sulfate and the solvents were removed under reduced pressure. Purification by flash column chromatography provided $\mathrm{NH}$-sulfoximine 4 .

(21) For HPLC analytical data, see the Supporting Information.

(22) For a recent illustrative example, see: Frings, M.; Thomé, I.; Schiffers, I.; Pan, F. F.; Bolm, C. Chem. Eur. J. 2014, 20, 1691.

(23) (a) Sedelmeier, J.; Bolm, C. J. Org. Chem. 2005, 70, 6904. (b) For a recent observation, see: Frings, M.; Atodiresei, I.; Bolm, C. Molbank 2014, M834.

(24) Schmidbaur, H.; Kammel, G. Chem. Ber. 1971, 104, 3234.

(25) For a recent study on sulfoximine N-alkylations under strongly basic conditions (with KOH in DMSO), see: Hendriks, C. M. M.; Bohmann, R. A.; Bohlem, M.; Bolm, C. Adv. Synth. Catal. 2014, 356, 1847.

(26) Meanwell, N. A. J. Med. Chem. 2011, 54, 2529.

(27) For early examples, see: García Mancheño, O.; Bolm, C. Org. Lett. 2007, 9, 2951. 